\title{
THE STABLE GROUP OF SELF-HOMOTOPY EQUIVALENCES OF SPHERE BUNDLES OVER THE SPHERE
}

\author{
By Seiya Sasao
}

\section{Introduction.}

The group of homotopy classes of homotopy equivalences of a $C W$-complex $X$ with itself, denoted here as $G(X)$, has been studied by various authors. Specially a stabilization of $G(X)$ is defined by the suspension homomorphism $G(X) \rightarrow$ $G(\Sigma X)$, denoted here as $G^{s}(X)=\lim _{N} G\left(\Sigma^{N} X\right)$, and has been studied by several authors $[3,4,5,6]$. Nevertheless, our examples for the computation of stable groups are not abundant. The purpose of this paper is to give a computation of the stable group $G^{s}(X)$ for sphere bundles over the sphere. If $X$ is a finite complex we can achieve $\lim _{N} G\left(\Sigma^{N} X\right)$ after a finite number of steps. Hence, it is sufficient for us to compute the group $G\left(\Sigma^{N} X\right)$ for a sufficiently large number $N$. The method is essentially based on Barcus-Barratt's theorem. However, we drive our main exact sequence from Puppe's sequence because our case is a stable one.

This is done in $\S 1$. In $\S 2$ some calculations which are needed in the later are done in the slightly more general situation. In $\S 3$ our theorem, stated as follows, is proved. Let $\xi$ be a $S^{p}$-bundle over $S^{q}$ and we denote by $K_{\xi}$ the total space of $\xi$. Let $\bar{\lambda}(\xi), \bar{J}(\xi)$ be the stable classes of $P_{*}$-image and $J$-image of the characteristic class of $\xi$ respectively, where $P_{*}: \pi_{q-1}(S O(p+1)) \rightarrow \pi_{q-1}\left(S^{p}\right), J$ : $\pi_{q-1}(S O(p+1)) \rightarrow \pi_{p+q}\left(S^{p+1}\right)$ are usual ones.

THEOREM A. Let $L$ be the complex $S^{p} \cup e^{q}$ which is obtained from attaching $q$-cell to $S^{p}$ by the $P_{* \text {-image }} \lambda(\xi)$. Then we have split extensions $(q>p+1)$ :

$$
\begin{aligned}
& 1 \longrightarrow \theta_{\xi} \longrightarrow G^{s}\left(K_{\xi}\right) \longrightarrow G^{s}(L) \longrightarrow 1 \quad \text { if } q \equiv 0 \bmod 4 \text { and } 2 \bar{J}(\xi) \neq 0, \\
& 1 \longrightarrow \theta_{\xi} \longrightarrow G^{s}\left(K_{\xi}\right) \longrightarrow G^{s}(L) \times Z_{2} \longrightarrow 1 \text { if } q \neq 0 \bmod 4,
\end{aligned}
$$

where $\theta_{\xi}$ is isomorphic to $\pi_{p+p+1}^{s}(\Sigma L)$ if $q \neq 0 \bmod 8$ and to $\pi_{p+q+1}^{s}(\Sigma L) / \bar{J}(\xi) \cdot \pi_{1}^{s}\left(S^{0}\right)$ if $q \equiv 0 \bmod 8$.

Further, about $G^{s}(L)$, we have

THEOREM B. There exist splat extensions $(q>p+1)$ :

Received January 29, 1980 


$$
\begin{aligned}
& 1 \longrightarrow \pi_{q-p}^{s}\left(S^{0}\right) / \bar{\lambda}(\xi) \cdot \pi_{1}^{s}\left(S^{0}\right) \longrightarrow G^{s}(L) \longrightarrow Z_{2} \times Z_{2} \longrightarrow 1 \\
& \text { if } 2 \xi=0 \text { and } p \text { is even }, \\
& 1 \longrightarrow \pi_{q-p}^{s}\left(S^{0}\right) / \bar{\lambda}(\xi) \cdot \pi_{1}^{s}\left(S^{0}\right) \longrightarrow G^{s}(L) \longrightarrow Z_{2} \longrightarrow 1 \\
& \text { if } 2 \bar{\lambda}(\xi) \neq 0 \text { and } p \text { is even } .
\end{aligned}
$$

\section{$\S 1$. Fundamental exact sequences}

Let $L$ be a 1-connected $C W$-complex and let $K$ be a complex of the form $L \bigcup_{\alpha} e^{n}(\operatorname{dim} L+2 \leqq n)$. Then as the Puppe's exact sequence we have the sequence

$$
\left[\Sigma^{2} L, \Sigma K\right] \underset{\Sigma^{2} \alpha}{\longrightarrow}\left[S^{n+1}, \Sigma K\right] \longrightarrow[\Sigma K, \Sigma K] \underset{R}{\longrightarrow}[\Sigma L, \Sigma L],
$$

where $R$ denotes the natural ring homomorphism induced by the restriction of maps. Since $G(\Sigma K)$ is the set consisting of invertible elements of $[\Sigma K, \Sigma K] R$ induces the homomorphism

$$
R \times O r: G(\Sigma K) \longrightarrow G(\Sigma L) \times Z_{2},
$$

where $\operatorname{Or}(f)$ is defined as the degree of $f: \Sigma K \rightarrow \Sigma K$ on the $(n+1)$-cell of $\Sigma K$.

LEMMA 1.1. The kernel of $R \times$ Or is isomorphic to

$$
\pi_{n+1}(\Sigma L) /\left[\Sigma^{2} L, \Sigma L\right] \cdot \Sigma^{2} \alpha \cup \Sigma \alpha \cdot \pi_{n+1}\left(S^{n}\right) .
$$

Proof. Suppose that $R \times O r(f)=(1,1)$ for $f$ of $[\Sigma K, \Sigma K]$. Then, in the sequence (I), we have that $1-f$ is contained in the image of $\left[S^{n+1}, \Sigma K\right]$. Hence we have

$$
f=1+\mu, \quad \mu \in \pi_{n+1}(\Sigma K) .
$$

Since $\operatorname{Or}(f)=\operatorname{Or}(1)+\operatorname{Or}(\mu)$ we obtain $\operatorname{Or}(\mu)=0$ and this means $\mu \in \pi_{n+1}(\Sigma L)$. Converesely if $\mu \in \pi_{n+1}(\Sigma L)$ it is clear that $1+\mu$ is contained in $G(\Sigma K)$ and $R \times$ $\operatorname{Or}(1+\mu)=(1,1)$. Thus the proof is completed from the diagram

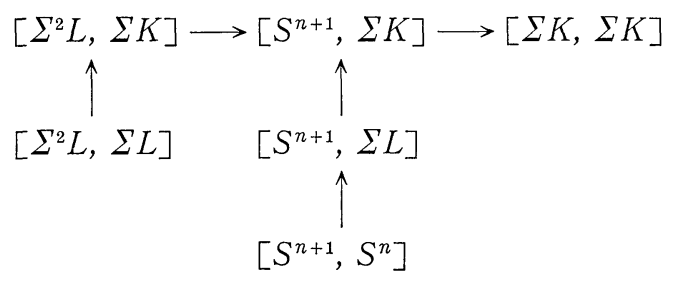

Example 1.2 (Theorem 3.13 of [8]). Let $K$ be a $C W$-complex $S^{m-1} \bigcup_{f} e^{n-1}$ $(m+2 \leqq n)$. Then we have two exact sequences: 
STABLE GROUP OF SELF-HOMOTOPY EQUIVALENCES

$$
\begin{array}{ll}
1 \longrightarrow H \longrightarrow G\left(S^{m} \bigcup_{\Sigma f} e^{n}\right) \longrightarrow Z_{2} \times Z_{2} \longrightarrow 1 & \text { if } 2 \Sigma f=0, \\
1 \longrightarrow H \longrightarrow G\left(S^{m} \bigcup_{\Sigma f} e^{n}\right) \longrightarrow Z_{2} \longrightarrow 1 & \text { if } 2 \Sigma f \neq 0 .
\end{array}
$$

where $H$ denotes $\pi_{n}\left(S^{m}\right) / \pi_{m+1}\left(S^{m}\right) \circ \Sigma^{2} f \cup \Sigma f \circ \pi_{n}\left(S^{n-1}\right)$.

Example 1.3. Let us consider the $N$-fold suspension

$$
\Sigma^{N}: G(\Sigma K) \longrightarrow G\left(\Sigma^{N+1} K\right) \quad(N \geqq 1)
$$

in the case of $K=S^{m-1} \bigcup_{f} e^{n-1}(m+2 \leqq n)$. From Example 1.2 we can obtain the following commutative diagram

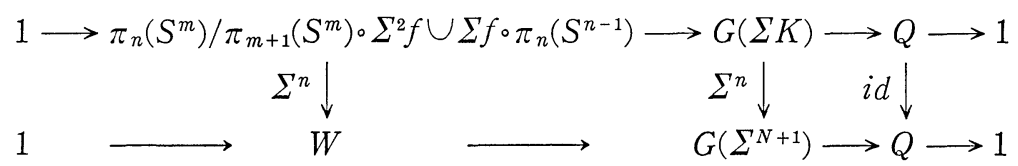

where $Q$ is $Z_{2} \times Z_{2}$ or $Z_{2}$ and $W$ denotes the group

$$
\pi_{n+N}\left(S^{m+N}\right) / \pi_{m+1+N}\left(S^{m+N}\right) \circ \Sigma^{N+2} f \cup \Sigma^{N+1} f \circ \pi_{n+N}\left(S^{n-1+N}\right) .
$$

Then, by using the standard argument, we have the exact sequence

$$
1 \longrightarrow W_{f} \longrightarrow G(\Sigma K) \stackrel{\Sigma}{\longrightarrow} G\left(\Sigma^{N+1} K\right) \longrightarrow \pi_{n+N}\left(S^{m+N}\right) / \Sigma^{N} \pi_{n}\left(S^{m}\right) \longrightarrow 1,
$$

where $W_{f}$ is the group $\Sigma^{-N}(0) / \Sigma^{-N}(0) \cap\left(\pi_{m+1}\left(S^{m}\right) \circ \Sigma^{2} f \cup \Sigma f \circ \pi_{n}\left(S^{n-1}\right)\right)$.

\section{$\S 2$. Some calculations}

Let $L_{1}$ and $L_{2}$ be $C W$-complexes $S^{p+1} \cup_{\alpha} e^{q+1}, S^{p+1} \bigcup_{\beta} e^{p+q+1}(q>p \geqq 2)$ respectively. Let $K$ be the $C W$-complex obtained from identifying the common sphere of $L_{1}$ and $L_{2}$. Our purpose of this section is to calculate $G\left(\Sigma^{N} K\right)$ for $N \geqq 2$. Since $\Sigma^{N} K$ has a $C W$-decomposition

$$
\Sigma^{N} K=S^{p+1+N} \bigcup_{\Sigma^{N} \alpha} e^{q+1+N} \bigcup_{\Sigma^{N} \beta} e^{p+q+1+N},
$$

we have the following exact sequence by applying Lemma 1.1 to the pair $\left(\Sigma^{N} K\right.$, $\left.L=\Sigma^{N} L_{1}\right)$

$$
1 \longrightarrow H_{\alpha, \beta} \longrightarrow G\left(\Sigma^{N} K\right) \longrightarrow G(L) \times Z_{2},
$$

where $H_{\alpha, \beta}=\pi_{N+p+q+1}(L) /[\Sigma L, L] \circ \Sigma^{N+1} \beta \cup \Sigma^{N} \beta \circ \pi_{N+p+q+1}\left(S^{N+p+q}\right)$.

LEMMA 2.1. If $N \geqq 2$ we have

$$
[\Sigma L, L] \circ \Sigma^{N+1} \beta \cup \Sigma^{N} \beta \circ \pi_{N+p+q+1}\left(S^{N+p+q}\right)=\Sigma^{N} \beta \circ \pi_{N+p+q+1}\left(S^{N+p+q}\right) .
$$


Proof. First we note a formula of [1] i. e.

$$
\Sigma^{b+1} x \circ \Sigma^{m+1} y=(-1)^{*} \Sigma^{a+1} y \circ \Sigma^{n+1} x
$$

for any $x \in \pi_{m+1}\left(S^{a+1}\right), y \in \pi_{n+1}\left(S^{b+1}\right)$.

Let $\eta: S^{3} \rightarrow S^{2}$ be the Hopf map. By the above formula we have

$$
\Sigma^{N+p-1} \eta \circ \Sigma^{N+1} \alpha=\Sigma^{N} \alpha \Sigma^{N+q-2} \eta \quad(N \geqq 2) .
$$

Hence, $\Sigma^{N+p-1} \eta$ is extendable to a map $\Sigma L \rightarrow L$ and the sub-group $[\Sigma L, L] \cdot \Sigma^{N+1} \beta$ is generated by the $i$-image of $\Sigma^{N+p-1} \eta^{\circ} \Sigma^{N+1} \beta\left(i: S^{N+p+1} \rightarrow L\right.$ is the inclusion). Thus the proof is completed by the formula

$$
\Sigma^{N+p-1} \eta \circ \Sigma^{N+1} \beta=\Sigma^{N} \beta \circ \Sigma^{N+p+q-2} \eta \quad(N \geqq 2) .
$$

LEMMA 2.2. The image of the homomorphism

$$
R \times O r: G\left(\Sigma^{N} K\right) \longrightarrow G(L) \times Z_{2}
$$

is equal to $G(L)$ if $2 \Sigma^{N} \beta \notin \Sigma^{N} \alpha \circ \pi_{N+p+q}\left(S^{N+q}\right)$ and to $G(L) \times Z_{2}$ if $2 \Sigma^{N} \beta \in$ $\Sigma^{N} \alpha \pi_{N+p+q}\left(S^{N+q}\right)$.

Proof. Since Hurewicz-image : $\pi_{*}\left(\Sigma^{N} K\right) \rightarrow H_{*}\left(\Sigma^{N} K\right)=Z(*=N+p+q+1)$ contains \pm 2 if and only if $2 \Sigma^{N} \beta=0$ in $\pi_{N+p+q}(L)$ the proof follows from the standard obstruction theory.

Next, consider the group $G(L)$. Then, from Example 1.2 and the formula $\Sigma^{N} \alpha \circ \Sigma^{N+q-2} \eta=\Sigma^{N+p-1} \eta \circ \Sigma^{N+1} \alpha(N \geqq 2)$ we can easily obtain

LEMMA 2.3. The following sequences are exact

$$
\begin{aligned}
1 \longrightarrow \pi_{N+q+1}\left(S^{N+p+1}\right) /\left\{\Sigma^{N} \alpha \circ \Sigma^{N+p-2} \eta\right\} \longrightarrow G(L) \longrightarrow Z_{2} \times Z_{2} \longrightarrow 1 \\
\text { if } 2 \Sigma^{N} \alpha=0 \\
1 \longrightarrow \pi_{N+q+1}\left(S^{N+p+1}\right) /\left\{\Sigma^{N} \alpha \circ \Sigma^{N+p-2} \eta\right\} \longrightarrow G(L) \longrightarrow Z_{2} \longrightarrow 1 \\
\text { if } 2 \Sigma^{N} \alpha \neq 0 .
\end{aligned}
$$

Now, from lemmas we obtain

PROPOSITION 2.4. If $N \geqq 2$ we have the exact sequence

$$
1 \longrightarrow H \longrightarrow G\left(\Sigma^{N} K\right) \longrightarrow \operatorname{Aut}\left(\bar{H}_{*}\left(\Sigma^{N} K\right)\right),
$$

where $H$ is a group-extension as follows $(*=N+p+q+1)$

$$
1 \longrightarrow \pi_{*}(L) /\left\{\Sigma^{N} \beta \circ \Sigma^{*-3} \eta\right\} \longrightarrow H \longrightarrow \pi_{N+q+1}\left(S^{N+p+1}\right) /\left\{\Sigma^{N} \alpha \circ \Sigma^{N+q-2} \eta\right\} \longrightarrow 1 .
$$

Moreover the image of $G\left(\Sigma^{N} K\right) \rightarrow$ Aut $\left(\bar{H}_{*}\left(\Sigma^{N} K\right)\right)$ is given as follows (we identify Aut $\left(\bar{H}_{*}\left(\Sigma^{N} K\right)\right)$ with $\left.Z_{2} \times Z_{2} \times Z_{2}\right)$ 
STABLE GROUP OF SELF-HOMOTOPY EQUIVALENCES

$$
\begin{array}{ll}
Z_{2} \times Z_{2} \times Z_{2} & \text { if } 2 \Sigma^{N} \alpha=0 \text { and } 2 \Sigma^{N} \beta \in \Sigma^{N} \alpha \circ \pi_{N+p+q}\left(S^{N+q}\right), \\
1 \times Z_{2} \times Z_{2} & \text { if } 2 \Sigma^{N} \alpha=0 \text { and } 2 \Sigma^{N} \beta \notin \Sigma^{N} \alpha \circ \pi_{N+p+q}\left(S^{N+q}\right), \\
Z_{2} \times 1 \times Z_{2} & \text { if } 2 \Sigma^{N} \alpha \neq 0 \text { and } 2 \Sigma^{N} \beta \in \Sigma^{N} \alpha \circ \pi_{N+p+q}\left(S^{N+q}\right), \\
1 \times 1 \times Z_{2} & \text { if } 2 \Sigma^{N} \alpha \neq 0 \text { and } 2 \Sigma^{N} \beta \notin \Sigma^{N} \alpha \circ \pi_{N+p+q}\left(S^{N+q}\right) .
\end{array}
$$

\section{$\S 3$. The case of sphere bundles over the sphere}

Let $\xi$ be a $S^{p}$-bundle over $S^{q}(q>p+1 \geqq 3)$. It is known in [7] that the total space $K_{\xi}$ has a $C W$-decomposition

$$
K_{\xi}=S^{p} \cup e^{q} \cup e^{p+q} .
$$

Let $T(\xi)$ be the Thom complex of the vector bundle associated with $\xi$. Since we may regard $T(\xi)$ as the mapping cone of the projection of $\xi$ (up to homotopy) there exists the natural map of degree 1

$$
C_{\xi}=T(\xi) \longrightarrow C_{\xi} / S^{q}=\Sigma\left(K_{\xi}\right) .
$$

Hence, by using the $C W$-decomposition of $T(\xi)$;

$$
T(\xi)=S^{p+1} \bigcup_{J(\xi)} e^{p+q+1},
$$

we have a $C W$-decomposition of $\Sigma K_{\xi}$ as follow

$$
\Sigma K_{\xi}=e^{p+q+1} \bigcup_{J(\xi)} S^{p+1} \bigcup_{\bar{\lambda}(\xi)} e^{q+1},
$$

where $J(\xi)$ is the usual notation and $\lambda(\xi)$ denotes the $P_{*}$-image $\left(P_{*}: \pi_{q-1}(S O(p+1))\right.$ $\left.\rightarrow \pi_{q-1}\left(S^{p}\right)\right)$.

Thus we can apply Prop. 2.4 to the case of $(K, L)=\left(K_{\xi}, L\right)$.

Let $\bar{J}(\xi)\left(\in \pi_{q-1}^{s}\left(S^{0}\right)\right), \bar{\lambda}(\xi) \quad\left(\in \pi_{q-p-1}^{s}\left(S^{0}\right)\right)$ be the stable classes of $J(\xi), \lambda(\xi)$ respectively. Then it is well known that

$$
\begin{aligned}
\bar{J}(\xi) & =0 \quad \text { if } q \equiv 3,5,6,7 & \bmod 8, \\
& \in Z_{2} \quad \text { if } q \equiv 1,2 & \bmod 8, \\
& \in Z_{m(q)} \quad \text { if } q \equiv 0 & \bmod 8
\end{aligned}
$$

and $\bar{J}(\xi) \circ \eta=0$ if $q \neq 0 \bmod 8, \neq 0$ if $q \equiv 0 \bmod 8$. Hence we have

Proposition 3.1. There exists following exact sequences:

$$
1 \longrightarrow \theta_{\xi} \longrightarrow G^{s}\left(K_{\xi}\right) \longrightarrow \operatorname{Aut}\left(\bar{H}_{*}\left(K_{\xi}\right)\right)
$$

and for $q \not \equiv 0 \bmod 8$

$$
1 \longrightarrow K_{\xi} \longrightarrow \theta_{\xi} \longrightarrow \pi_{q-p}^{s}\left(S^{0}\right) /\{\bar{\lambda}(\xi) \circ \eta\} \longrightarrow 1,
$$




$$
1 \longrightarrow \pi_{q}^{s}\left(S^{0}\right) /\left\{\bar{\lambda}(\xi) \circ \pi_{p+1}^{s}\left(S^{0}\right)\right\} \longrightarrow K_{\xi} \longrightarrow\left\{x \mid x \in \pi_{q}^{s}\left(S^{0}\right), \bar{\lambda}(\xi) \circ x=0\right\} \longrightarrow 1 .
$$

Next, Let consider the splitting of group-extensions which are already obtained in $\S 2$.

LEMMA 3.2. There exists an involution $h_{\xi}: \Sigma^{N} T(\xi) \rightarrow \Sigma^{N} T(\xi)$ which is degree -1 on $S^{N+p+1}(N \geqq 2)$.

Proof. Since we may consider $K_{\xi}$ as $\left(S^{p} \times E_{-q}^{q}\right) \cup\left(S^{p} \times E_{+}^{q}\right)$ we have the map $k_{\xi}: K_{\xi} \rightarrow K_{\xi}$ defined by

$$
k_{\xi}\left(v, x_{\varepsilon}\right)=\left(-v, x_{\varepsilon}\right) \quad\left(\varepsilon= \pm, x \in E_{\varepsilon}^{q}\right) .
$$

Then $k_{\xi}$ induces an involution $T\left(k_{\xi}\right): T(\xi) \rightarrow T(\xi)$ of degree $(-1)^{p+1}$ on $S^{N+p+1}$. Hence, it is sufficient to take $h_{\xi}$ as the suspension of $T\left(k_{\xi}\right)$ for even $p$ and of $T\left(K_{\xi \oplus 1}\right)$ for odd $p$.

Remark. We note that $k_{\xi}$ is commutative with any bundle map, i. e. we have a commutative diagram:

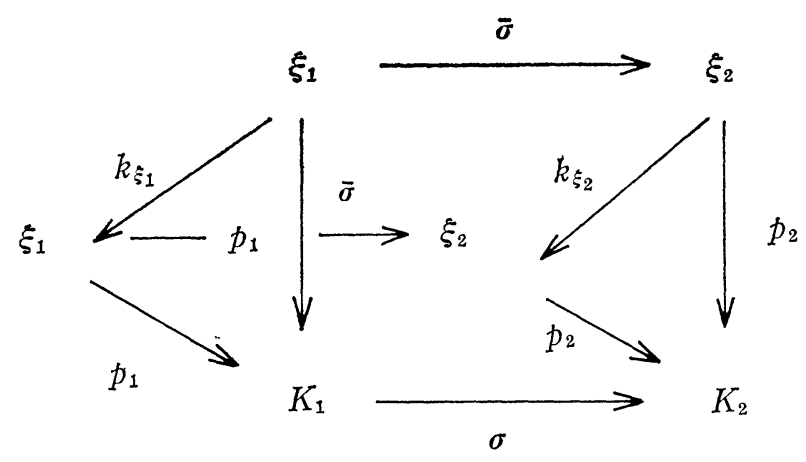

Next, for any homotopy equivalence $f: L \rightarrow L$, define $\bar{f}: \Sigma^{N} K \rightarrow \Sigma^{N} K$ by

$$
\bar{f} \mid L=f, \text { and } \bar{f} \mid \Sigma^{N} T(\xi)=h_{\xi}^{(3+\operatorname{deg} f) / 2},
$$

where $\operatorname{deg} f$ is the degree on $S^{N+p+1} \subset L$.

LEMmA 3.3. Define $S: G(L) \rightarrow G\left(\Sigma^{N} K_{\xi}\right)$ by $S(f)=\bar{f}$. Then, $S$ is a homomorphism and the composite $R \circ S$ is the identity.

Proof. For $f, g: L \rightarrow L$, we have

and

$$
f \circ g|L=f \circ g| L=f|L \circ g| L
$$

$$
\begin{aligned}
& f \circ g \mid \Sigma^{N} T\left(K_{\xi}\right)=h_{\xi}^{(3+\operatorname{deg} f g) / 2}=h_{\xi}^{\left(3+\operatorname{deg} f^{\circ} \operatorname{deg} g\right) / 2}
\end{aligned}
$$

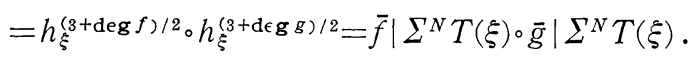


LEMMA 3.4. If $q \neq \equiv 0 \bmod 4$ there exists and involution (up to homotopy) $l_{\xi}$ : $\Sigma^{N} T(\xi) \rightarrow \Sigma^{N} T(\xi)$ (sufficiently large $N$ ) which is of degree 1 on $S^{N+p+1}$ and of degree -1 on $e^{N+p+q+1}$, and further we have $h_{\xi^{\circ}} l_{\xi}=l_{\xi} \circ h_{\xi}$.

Proof. First we note the following sub-lemma

Sub-Lemma. Let $X=S^{N} \bigcup_{\boldsymbol{\sigma}} e^{N+p}$ be a $C W$-complex ( $N$ : sufficiently large). Let $f: X \rightarrow X$ be a homotopy equivalence with degree 1 on $S^{N}$ and degree -1 on $e^{N+p}$. Then the composite $f \circ f$ is the identity in $[X, X]$.

For, from Puppe's sequence

$$
\left[S^{N+p}, X\right] \longrightarrow[X, X] \longrightarrow\left[S^{N}, X\right]
$$

there exists an element $\alpha \in \pi_{N+p}(X)$ such that $f=1_{X}+\alpha$. Hence, we have $f \circ f=$ $\left(1_{X}+\alpha\right)\left(1_{X}+\alpha\right)=1_{X}+2 \alpha+(\operatorname{deg} \alpha) \alpha$. On the other hand, since degree $f=1+$ degree $\alpha$, we have degree $\alpha=-2$. Thus the proof is complete.

Secondly we prove Lemma 3.4. Since $2 \xi=0$ in the stable range the bundle induced by a map: $S^{q} \rightarrow S^{q}$ of degree -1 is equivalent to $\xi$. Hence, from sublemma, we have a bundle map: $K_{\xi} \rightarrow K_{\xi}$ which induces the map $l_{\xi}$, as desired. The commutativity follows from the above remark.

For $q \not \equiv 0 \bmod 4$, we define an involution $\bar{l}_{\hat{\xi}}: \Sigma^{N} K \rightarrow \Sigma^{N} K$ by

$$
i_{\xi} \mid L=l_{L} \quad \text { and } \quad i_{\xi} \mid \Sigma^{N} T(\xi)=l_{\xi} .
$$

Now, consider the correspondence $V: G^{s}(L) \times Z_{2} \rightarrow G^{s}(K)$ defined by

$$
V(f, \varepsilon)=i_{\xi}^{*} \circ S(f)=i_{\xi}^{*} \circ \bar{f},
$$

where $*$ denotes $(\varepsilon+3 \operatorname{deg} f) / 2$ and $\varepsilon$ is 1 or -1 .

LEMMA 3.5. $V$ is a homomorphism and the composite $R \times O r \cdot V$ is the identity.

Proof. The former follows from the commutativity of Lemma 3.4 and the latter is easily obtained from the definitions.

Now the theorem stated in the introduction is an easy consequence from Lemmas 1.1, 2.2, 3.4 and 3.5.

Example 3.6. Let $\xi$ be the trivial bundle, hence $K_{\xi}=S^{p} \times S^{q}(q>p \geqq 2)$. Then we have a split extension

$$
1 \longrightarrow \pi_{q}^{s}\left(S^{0}\right) \oplus \pi_{p}^{s}\left(S^{0}\right) \longrightarrow G^{s}\left(K_{\xi}\right) \longrightarrow G \times Z_{2} \longrightarrow 1,
$$

where $G$ denotes the group $\left\{\left(\begin{array}{cc}\varepsilon_{2} & \gamma \\ 0 & \varepsilon_{1}\end{array}\right), \gamma \in \pi_{q-p}^{s}\left(S^{0}\right), \varepsilon_{\imath}=1\right.$ or -1$\}$.

Further, $G^{s}\left(K_{\xi}\right)$ is isomorphid to the group 


$$
\left\{\left(\begin{array}{ccc}
\varepsilon_{3} & \alpha & \beta \\
0 & \varepsilon_{2} & \gamma \\
0 & 0 & \varepsilon_{1}
\end{array}\right), \alpha \in \pi_{p}^{s}\left(S^{0}\right), \beta \in \pi_{q}^{s}\left(S^{0}\right), \gamma \in \pi_{q-p}^{s}\left(S^{0}\right), \varepsilon_{2}=1,-1\right\} .
$$

Example 3.7. Let $W_{n, 2}$ be the Stiefel manifold $U(n) / U(n-2)$ and let $\xi_{n}$ be the sphere bundle $S^{2 n-3} \rightarrow W_{n, 2} \rightarrow S^{2 n-1}$. Then it is well known that $\Sigma^{2} J\left(\xi_{n}\right)=0$ (1.17 of [7]) and $\lambda\left(\xi_{n}\right)=0$ if $n$ is even, $=\eta\left(\in \pi_{1}^{s}\left(S^{0}\right)\right)$ if $n$ is odd. Thus if $n$ is even the case reduces to Example 3.6, namely $K_{\xi_{n}}$ is stablly equivalent to $S^{p} \times S^{q}$ (up to homotopy). Next, let $C P^{2}$ be the complex projective plane. Then, for odd $n$, we have a split extension:

$$
1 \longrightarrow \pi_{4 n-4}^{s}\left(C P^{2}\right) \longrightarrow G^{s}\left(W_{n, 2}\right) \longrightarrow Z_{2} \times Z_{2} \times Z_{2} \longrightarrow 1 \text {. }
$$

\section{REFERENCES}

[1] M. G. Barratt and P. J. Hilton, On join operation in homotopy group, Proc. Lond. Math. Soc. (3) 3 (1953), 430-445.

[2] W. B. Barcus AND M. G. Barratt, On the homotopy classification of a fixed map, Trans. Amer. Math. Soc., 33 (1958), 57-74.

[3] P. T. Johnston, The stable group of homotopy equivalences, Quat. J. Math. Oxford, (2) 23 (1972), 213-219.

[4] D.W. KAHN, The group of stable self-equivalences, Topology., vol 11 (1972), 133-140.

[5] D.W. KAHN, Realization problems for the group of homotopy classes of selfequivalences, Math. Annalen, 220 (1976), 37-46.

[6] A. J. SieRAdski, Stabilization of self-equivalences of psuedo-projective spaces, Michigan Math. J., 19 (1972), 109-119.

[7] I. M. JAmes AND J.H.C. WhiteheAd, The homotopy theory of sphere bundles over spheres I, Proc. Lond. Math. Soc. (3), 5 (1954), 196-218.

[8] S. OKa, N. Sawashita and M. Sugawara, On the group of self-equivalences of a mapping cone, Hiroshima Math. J., 4 (1974), 9-28.

Dept. OF MATH.

TOKYO INST. OF TECHNOLOGY

Oh-Okayama Meguro-ku

TOKYO, JAPAN. 